\title{
Reflection images from ambient seismic noise
}

\section{Deyan Draganov ${ }^{1}$, Xander Campman $^{2}$, Jan Thorbecke $^{1}$, Arie Verdel ${ }^{2}$, and Kees Wapenaar ${ }^{1}$}

\begin{abstract}
One application of seismic interferometry is to retrieve the impulse response (Green's function) from crosscorrelation of ambient seismic noise. Various researchers show results for retrieving the surface-wave part of the Green's function. However, reflection retrieval has proven more challenging. We crosscorrelate ambient seismic noise, recorded along eight parallel lines in the Sirte basin east of Ajdabeya, Libya, to obtain shot gathers that contain reflections. We take advantage of geophone groups to suppress part of the undesired surface-wave noise and apply frequency-wavenumber filtering before crosscorrelation to suppress surface waves further. After comparing the retrieved results with data from an active seismic exploration survey along the same lines, we use the retrieved reflection data to obtain a migrated reflection image of the subsurface.
\end{abstract}

\section{INTRODUCTION}

Since the beginning of this century, seismic interferometry has rapidly become popular in a variety of applications (for an extensive overview, see Wapenaar et al. 2008 and Schuster 2009). One of the most intriguing applications of the method is retrieving the seismic impulse response (Green's function) from crosscorrelation of diffuse wavefields, where the diffuse fields can result in a closed system from random multiple scattering inside the system or from the system's boundaries (Weaver and Lobkis, 2001) or in an open system from a random distribution of noise sources, e.g., ambient seismic noise (Campillo and Paul, 2003).

The earth is a closed system, but the wavefield is usually far from diffuse. The diffusivity depends on observation points, season, duration of observation, complexity of the medium, frequencies of interest, and wave type (e.g., Okada, 2003; Stehly et al., 2006; Pedersen et al., 2007; Picozzi et al., 2009). At smaller scales, an extra complication is that the encompassing system is not closed. Heuristic arguments using the fundamental properties of reciprocity and time-reversal invariance (Derode et al., 2003) and stationary-phase analysis of the crosscorrelation integral (Snieder, 2004) have been used to explain that in the presence of enough random sources and/or scatterers, Green's function retrieval by crosscorrelation is possible in open systems. Researchers have been able to retrieve the surface-wave part of the Green's function from ambient-noise correlations (e.g., Shapiro and Campillo, 2004; Sabra et al., 2005a). This information has been used for regional tomographic imaging (Sabra et al., 2005b; Shapiro et al., 2005) and monitoring changes in volcanic interiors (Sens-Schönfelder and Wegler, 2006; Brenguier et al., 2008).

Seismic surface-wave retrieval is quite robust, which may be explained by the fact that surface waves are the strongest events in the ambient noise. Nevertheless, the resolution power of surface-wave data in the depth direction usually is insufficient for seismic exploration. It is much more desirable to retrieve reflection data, which allow the extraction of velocity information and construction of depth images with higher resolution.

A classic analysis shows that the 1D depth-dependent variations resulting from the earth's layering can be resolved from the autocorrelation of the transmission response of a deeply buried noise source (Claerbout, 1968). In the first decades after this idea was conceived, applications were rare (Scherbaum, 1987a, 1987b; Daneshvar et al., 1995). Using the Green's theorem together with signal-processing basics, the 1D method was extended for a 3D layered earth (Wapenaar et al., 2002) and subsequently generalized to a theory that describes recovery of the full 3D Green's function (surface waves and reflected waves) from noise correlations (Wapenaar, 2004).

The latter theory shows that, for observation points at the earth's surface, the surface-wave part of the Green's function is retrieved from ambient-noise sources located at or near the surface, whereas reflection-response retrieval requires a distribution of noise sources in the depths below the receivers (see Ravi Kumar and Bostock, 2006, for an example with teleseismic arrivals, i.e., transient sources, in a $1 \mathrm{D}$ medium). Reflected waves from noise are more challeng-

Manuscript received by the Editor 1 February 2009; revised manuscript received 20 March 2009; published online 22 September 2009.

${ }^{1}$ Delft University of Technology, Department of Geotechnology, Delft, The Netherlands. E-mail: d.s.draganov@tudelft.nl; j.w.thorbecke@tudelft.nl; c.p.a.wapenaar@tudelft.nl.

${ }^{2}$ Shell International Exploration and Production B.V, Rijswijk, The Netherlands. E-mail: xander.campman@ shell.com; arie.verdel@shell.com.

(C) 2009 Society of Exploration Geophysicists. All rights reserved. 
ing to retrieve than surface waves because reflection amplitudes decay more rapidly with distance and the demands on the distribution of the ambient-noise sources are more severe. In general, surfacewave noise drowns out the subtle body-wave noise required for imaging subsurface structures with high resolution.

Nevertheless, there is evidence that reflections can be retrieved (Draganov et al., 2007). In the following, we show how we obtain body-wave reflections from behind the masquerading surface-wave noise and use this information to retrieve reflection profiles and a pseudo-3D reflection image of the earth's subsurface. Retrieval of reflections from ambient seismic noise may have applications in exploration in frontier regions, in areas with difficult terrain conditions, in naturally sensitive areas, in permanent monitoring for reservoir production, and in $\mathrm{CO}_{2}$ sequestration surveillance.

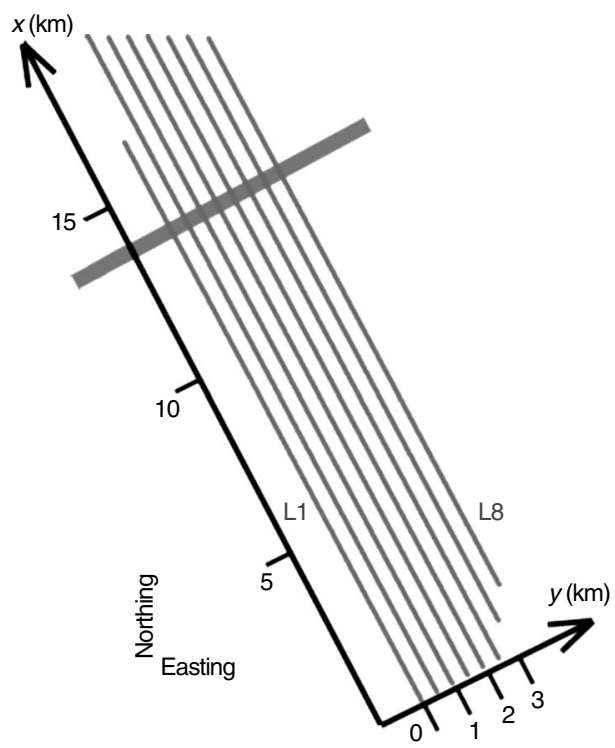

Figure 1. Geometry of the field survey. The thin gray lines represent the geophone lines; the thick gray line indicates a traffic road intersecting the survey lines.

a) Horizontal distance $(\mathrm{km})$

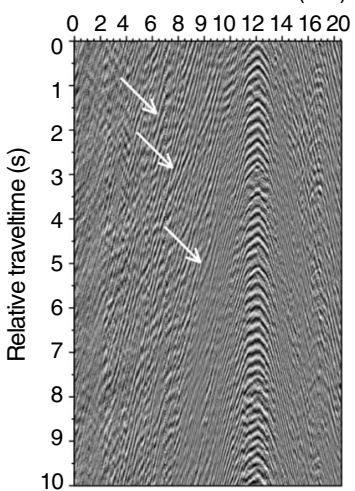

b)

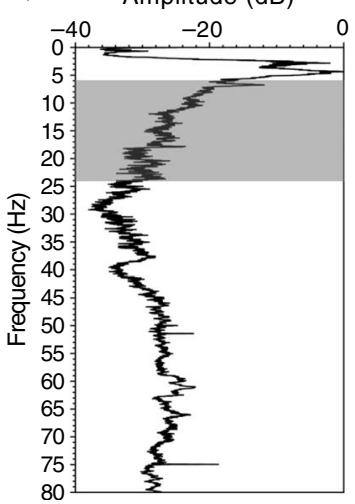

c) Horizontal distance $(\mathrm{km})$ 0246891012141620

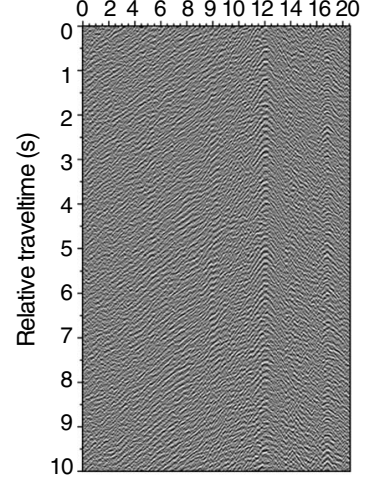

Figure 2. (a) First $10 \mathrm{~s}$ from an arbitrary ambient-noise record. The white pointers indicate surface waves. (b) Amplitude spectrum of the panel in (a) after summing over all traces. (c) The panel in (a) after frequency-wavenumber and band-pass filtering.

\section{FIELD GEOMETRY AND INITIAL PROCESSING}

Data were acquired by Shell in the northeastern part of the Sirte basin, east of Ajdabeya, Libya. The acquisition geometry consisted of eight parallel lines (L1-L8) of about $20 \mathrm{~km}$ with a separation distance of $500 \mathrm{~m}$ (Figure 1). Each line consisted of approximately 400 receiver channels placed $50 \mathrm{~m}$ apart. Each receiver channel represented the center of a group of 48 vertical-component geophones. The geophone patterns were designed such that surface waves with the most dominant wavenumbers were suppressed in inline and crossline directions. To maximize the amount of registered energy from the presumed subsurface sources, it is best to record for a long time. In our case, noise was recorded for an approximately 11-hour period. Because of restrictions imposed by the recording equipment, the data were stored in about 900 time windows of $47 \mathrm{~s}$ (ambientnoise records). Figure $2 \mathrm{a}$ shows the first $10 \mathrm{~s}$ from an arbitrary ambient-noise record along one of the lines. This recording is dominated by strong remnants of spatially coherent surface waves that were not suppressed by the geophone groups and were excited randomly in time by traffic along a road bisecting the survey around $14 \mathrm{~km}$ at its northern section (see Figure 1).

When we retrieve reflections, we want to suppress surface waves as much as possible. Frequency and frequency-wavenumber analysis reveals that the most energetic part of the surface waves is concentrated mainly below $6 \mathrm{~Hz}$. Furthermore, the frequency spectrum in Figure $2 b$ shows significant spectral notches and peaks above $24 \mathrm{~Hz}$, which may complicate our analysis. Therefore, for further processing, we select the energy between 6 and $24 \mathrm{~Hz}$ with a bandpass filter.

Next, striving to bring forward possible retrieved reflections and minimize cross-terms between surface waves and body waves, we remove the remaining direct inline surface waves, traveling with an approximately constant velocity in this frequency band, with a frequency-wavenumber filter. Figure $2 \mathrm{c}$ shows the panel from Figure $2 \mathrm{a}$ after filtering. The surface-wave energy has been reduced significantly. To ensure that each ambient-noise record contributes equally when retrieving the Green's function, we energy normalize each trace in each ambient-noise record.

\section{RETRIEVAL OF REFLECTION PROFILES AND A PSEUDO-3D IMAGE}

We apply seismic interferometry to the recorded ambient noise using the relation (Wapenaar, 2004)

$$
\begin{gathered}
\left\{G_{p, q}\left(\mathbf{x}_{\mathcal{A}}, \mathbf{x}_{\mathcal{B}}, t\right)+G_{p, q}\left(\mathbf{x}_{\mathcal{A}}, \mathbf{x}_{\mathcal{B}},-t\right)\right\} * a(t) \\
\approx \sum_{i}^{N} \nu_{p}^{i}\left(\mathbf{x}_{\mathcal{A}},-t\right) * \nu_{q}^{i}\left(\mathbf{x}_{\mathcal{B}}, t\right) .
\end{gathered}
$$

Here, $G_{p, q}\left(\mathbf{x}_{\mathcal{A}}, \mathbf{x}_{\mathcal{B}}, \pm t\right)$ denote the causal and timereversed Green's tensors between positions $\mathbf{x}_{\mathcal{B}}$ and $\mathbf{x}_{\mathcal{A}}, t$ denotes time, $\nu_{p(q)}^{i}$ represents the component of the particle velocity recorded in the $x_{p(q)}$ direction $(p, q=1,2,3)$ in the $i$ th ambient-noise record, $a(t)$ stands for the autocorrelation of the source time function of the noise sources, and the 
asterisk denotes convolution. The sum on the right-hand side is carried out over all $N$ available ambient-noise records. The relation is derived assuming that the noise sources are uncorrelated and illuminate the observation points from all directions and that the recorded noise can be described as an ergodic random process.

Relation 1 states that the sum over time of crosscorrelations of noise records at a receiver pair approximates the sum of the causal and time-reversed Green's function between the two receivers, convolved with the autocorrelation of the noise. The Green's function includes the desired reflection response. Thus, to retrieve the reflection response for a virtual source position, we fix one trace at a time (fixed $\mathbf{x}_{\mathcal{B}}$ ) and correlate this trace with all other traces from one receiver line (variable $\mathbf{x}_{\mathcal{A}}$ ) to obtain a correlation panel. Repeating this process for all $N \approx 900$ ambient-noise records from the chosen line and summing the resulting $N$ correlation panels, we obtain the lefthand side of equation 1 . To extract the Green's function from this result, we must remove the imprint of the autocorrelation of the source time function $a(t)$. This is done by deconvolving with a wavelet extracted from the autocorrelation trace using a narrow window around zero time.

The ambient-noise sources in the subsurface are not optimally (homogeneously) distributed, so some parts of the Green's function are retrieved only in the causal part of the correlation result and others in the acausal part. To obtain a more accurate Green's function, the retrieved time-advanced and time-retarded parts are summed. The final result is a retrieved common-shot gather. It is the response of a virtual source at $\mathbf{x}_{\mathcal{B}}$ observed by receivers at $\mathbf{x}_{\mathcal{A}}$.

Figure 3 a shows a retrieved common-shot gather along line L4 for a virtual source located at $1 \mathrm{~km}$. We observe approximately hyperbolic events associated with reflections around $0.15,0.3$, and $0.4 \mathrm{~s}$, as well as several events between 0.7 and $1.1 \mathrm{~s}$. The same reflection events can be observed in an active-source common-shot gather, where the vibroseis source has been placed at approximately the same location (Figure 3b highlighted in red). To make the comparison easier, the shot gather in Figure $3 \mathrm{~b}$ is shown after frequency-wavenumber filtering to remove some remnant surface waves. Figure $3 \mathrm{a}$ and $\mathrm{b}$ is further band-pass filtered between 9 and $18 \mathrm{~Hz}$ to equalize the frequency contents. Still, we can see that the reflections in the active-source gather result from waves with higher frequencies.

If the body waves in the recorded noise have lower frequencies, the resulting retrieved reflections will exhibit the same lower frequencies. Some linear inclined events can be seen on the retrieved common-shot gather. These events can be a result of aliasing (compare to similar events in the active-source gather), but they also may, in part, be a result of correlation of road-traffic surface waves propagating in directions different from the inline direction. If surface waves have propagated in such directions, the inline frequency-wavenumber $(f-k)$ filter that we use will not remove them.

We repeat the crosscorrelation procedure by choosing the virtual-source position at every receiver channel along each of the eight lines, retrieving about 3200 shot gathers. The obtained

a)

b) virtual reflection data allow us to construct an image of the subsurface. The large separation between the lines $(500 \mathrm{~m})$ does not allow the use of a full 3D migration procedure. Instead, we process each line separately using 2D schemes.

First, we obtain poststack time-migrated images of the subsurface (see Figure 4a) by following a standard processing scheme consisting of statics correction (to a mean sea level), common-midpoint sorting, interactive velocity analysis, normal-moveout correction, stacking, and phase-shift time migration (Claerbout, 1985; Yilmaz, 1999). From a priori information, we know that the subsurface in this

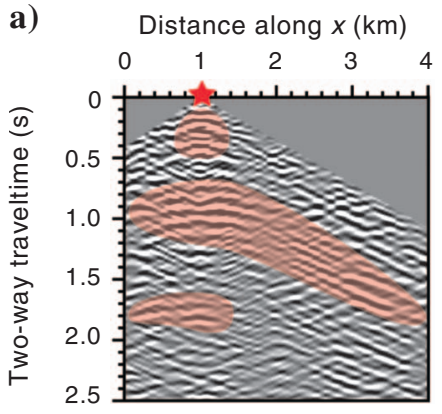

b) Distance along $x(\mathrm{~km})$

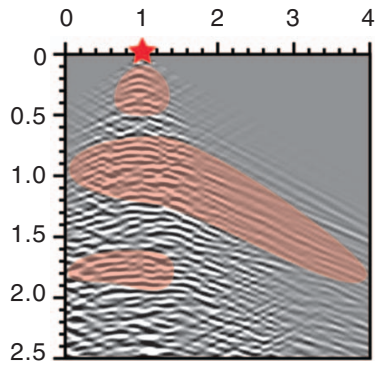

Figure 3. (a) Retrieved common-shot gather from the ambient noise. (b) Active-source common-shot gather recorded at the same location. The red stars indicate the positions of the virtual and real sources. Sections with coinciding events are highlighted in transparent red. Events at times earlier than the direct-wave arrival have been muted.
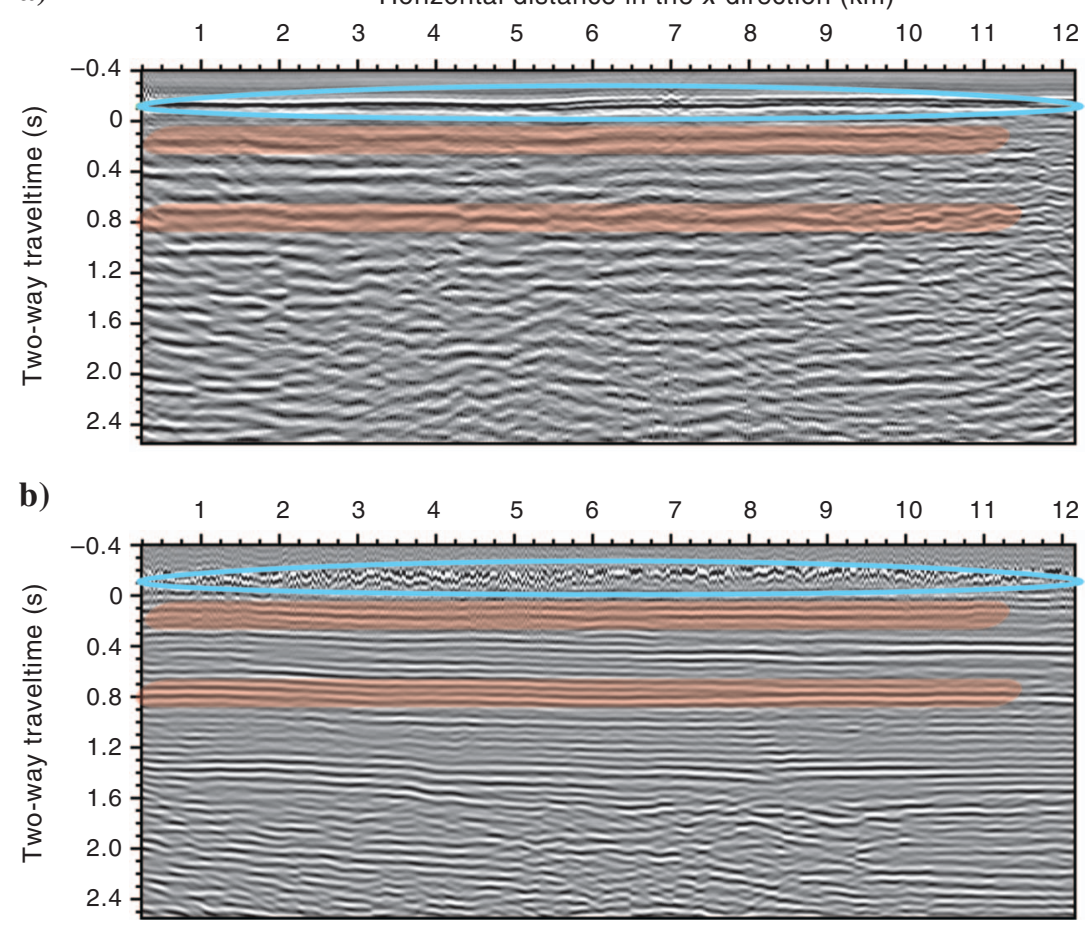

Figure 4. Poststack time-migrated sections along the first $12 \mathrm{~km}$ of line L4 from Figure 1 obtained from (a) the retrieved shot gathers and (b) the active data. Coinciding imaged reflectors are highlighted in transparent red. The blue ellipses indicate the earth's surface. Zero time refers to mean sea level. 


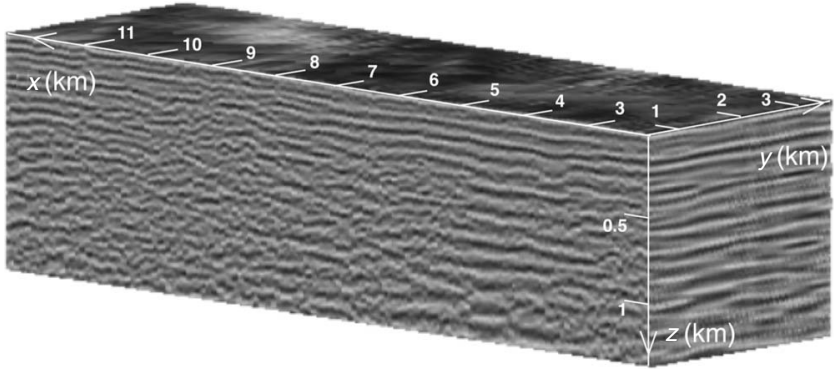

Figure 5. A pseudo-3D image giving an impression of the subsurface below the survey lines. The pseudo-3D image from Figure 5 played along the $y$-axis starting from line L1. Figure enhanced online. [DOI: http://dx.doi.org/10.1190/1.3193529.1]

area exhibits mild lateral variations of the seismic properties. The velocities are estimated at every $500 \mathrm{~m}$ along each line from the reflection events in the retrieved data, using a standard velocity-analysis method (Yilmaz, 1999).

To establish the robustness of the retrieved reflections, we compare the retrieved time-migrated results to results from the active survey (Figure $4 \mathrm{~b}$ ). The active-source image has been band-pass filtered to try to match the frequency content of the retrieved data (hence, the seemingly discontinuous line of the earth's surface). Nevertheless, the amplitude spectra of the two results are different, which might come from the generally lower-frequency character of the retrieved shot gathers.

Comparing the two time-migrated sections, in particular, we observe two shallow marker events around 0.1 and $0.8 \mathrm{~s}$. These reflection events coincide in both images. In addition, there are several other coinciding coherent events. The apparent jump of the shallowest marker event at horizontal distance around $x=3 \mathrm{~km}$ might be caused by incorrect velocity picking. At times beyond $1 \mathrm{~s}$, events in the passive image lose their spatial coherence and cannot be identified unambiguously with reflections in the active image. Thus, as we would expect, it seems harder to extract deeper reflections because of the larger geometric spreading of body waves. Having longer records of the ambient seismic noise (days, weeks) might solve this problem because potentially we would record waves from more subsurface sources and consequently improve the stacking power and illumination of the ambient-noise data.

Even though during the velocity analysis we picked velocities such that energy from multiple reflection paths is suppressed, there appears to be some remnant multiple energy in the left part of the image from the ambient-noise data in Figure $4 \mathrm{a}$ around $1 \mathrm{~s}$. This event is absent in the active image (Figure $4 \mathrm{~b}$ ) where an additional processing step was applied to suppress multiple reflections. We show the comparison until $12.15 \mathrm{~km}$ along the line. No coherent events could be seen at locations closer to the traffic road around $14 \mathrm{~km}$. Despite our best efforts, strong surface-wave remnants remain at these locations, obscuring possible retrieved reflections and consequently making velocity picking very hard.

Having validated the authenticity of the retrieved shallow marker events, we proceed to obtain structural information. For this purpose, we apply $2 \mathrm{D}$ prestack depth migration to the retrieved shot records along the eight lines. We use a constant-gradient velocity model as a first approximation of the picked velocities from the retrieved data. The eight depth-migrated sections are interpolated linearly to give an impression of the 3D structure of the earth below the surveyed area (see Figure 5). We can appreciate several imaged re- flectors, e.g., the reflectors at $0.18,0.4$, and $0.55 \mathrm{~km}$. The ancillary online video follows the imaged reflectors in $x$ - $z$ profiles along the $y$-axis in Figure 5, starting from line L1.

\section{CONCLUSIONS}

We have applied seismic interferometry to ambient seismic noise recorded along eight lines in a desert area in Libya and correlated about 11 hours of noise to retrieve virtual shot gathers. These shot gathers, compared with shot gathers from active data recorded with sources located at the same positions, reveal several correctly retrieved reflections. The retrieved shot gathers, processed using a standard seismic processing flow to obtain poststack time-migrated reflection images, were validated against a poststack time-migrated image obtained from the active data. Good agreement between them was found for two-way traveltimes up to $1 \mathrm{~s}$. Furthermore, we prestack depth-migrated the retrieved shot gathers to construct a pseudo-3D depth image of the subsurface below the eight lines. Retrieval of reflection images from ambient seismic noise has potential for seismic exploration, reservoir production monitoring, and $\mathrm{CO}_{2}$ sequestration surveillance.

\section{ACKNOWLEDGMENTS}

The research of D. Draganov was sponsored by the Technology Foundation STW, applied science division of NWO (project 08115), and by Shell International Exploration \& Production B.V. We thank the Libyan National Oil Company for permission to publish these results and Shell in Libya (especially Erik Kleiss, Rian de Jong, Mark Peach and Alan Smith) for collecting and making available the passive data. We thank Dirk-Jan van Manen and two anonymous reviewers for their constructive comments.

\section{REFERENCES}

Brenguier, F., N. M. Shapiro, M. Campillo, V. Ferrazzini, Z. Duputel, O. Coutant, and A. Nercessian, 2008, Towards forecasting volcanic eruptions using seismic noise: Nature Geoscience, 1, 126-130.

Campillo, M., and A. Paul, 2003, Long-range correlations in the diffuse seismic coda: Science, 299, 547-549.

Claerbout, J. F., 1968, Synthesis of a layered medium from its acoustic transmission response: Geophysics, 33, 264-269.

, 1985, Imaging the earth's interior: Blackwell Scientific Publications, Inc.

Daneshvar, M. R., C. S. Clarence, and M. K. Savage, 1995, Passive seismic imaging using microearthquakes: Geophysics, 60, 1178-1186.

Derode, A., E. Larose, M. Tanter, J. de Rosny, A. Tourin, M. Campillo, and M. Fink, 2003, Recovering the Green's function from field-field correlations in an open scattering medium: Journal of the Acoustic Society of America, 113, 2973-2976.

Draganov, D., K. Wapenaar, W. Mulder, J. Singer, and A. Verdel, 2007, Retrieval of reflections from seismic background-noise measurements: Geophysical Research Letters, 34, L04305.

Okada, H., ed., 2003, The microtremor survey method: SEG.

Pedersen, H. A., F. Krüger, and the Svekalapko Seismic Tomography Working Group, 2007, Influence of the seismic noise characteristics on noise correlations in the Baltic shield: Geophysical Journal International, 168, 197-210.

Picozzi, M., S. Parolai, D. Bindi, and A. Strollo, 2009, Characterization of shallow geology by high-frequency seismic noise tomography: Geophysical Journal International, 176, 164-174.

Ravi Kumar M., and M. G. Bostock, 2006, Transmission to reflection transformation of teleseismic wavefields: Journal of Geophysical Research, 111, B08306.

Sabra, K. G., P. Gerstoft, P. Roux, W. A. Kuperman, and M. C. Fehler, 2005a, Extracting time-domain Green's function estimates from ambient seismic noise: Geophysical Research Letters, 32, L03310.

, 2005b, Surface wave tomography from microseisms in southern Cali- 
fornia: Geophysical Research Letters, 32, L143111.

Scherbaum, F., 1987a, Seismic imaging of the site response using microearthquake recordings, Part I — Method: Bulletin of the Seismological Society of America, 77, 1905-1923.

, 1987b, Seismic imaging of the site response using microearthquake recordings, Part II - Application to the Swabian Jura, southwest Germany, seismic network: Bulletin of the Seismological Society of America, 77, $1924-1944$.

Schuster, G. T., 2009, Seismic interferometry: Cambridge University Press. Sens-Schönfelder, C., and U. Wegler, 2006, Passive image interferometry and seasonal variations of seismic velocities at Merapi volcano, Indonesia: Geophysical Research Letters, 33, L21302.

Shapiro, N. M., and M. Campillo, 2004, Emergence of broadband Rayleigh waves from correlations of the ambient seismic noise: Geophysical Research Letters, 31, L07614.

Shapiro, N. M., M. Campillo, L. Stehly, and M. H. Ritzwoller, 2005, Highresolution surface wave tomography from ambient seismic noise: Science, 307, 1615-1618.
Snieder, R., 2004, Extracting the Green's function from the correlation of coda waves: A derivation based on stationary phase: Physical Review E, 69, 046610

Stehly, L., M. Campillo, and N. M. Shapiro, 2006, A study of the seismic noise from its long-range correlation properties: Journal of Geophysical Research, 111, B10306.

Wapenaar, K., 2004, Retrieving the elastodynamic Green's function of an arbitrary inhomogeneous medium by cross-correlation: Physical Review Letters, 93, 254301.

Wapenaar, K., D. Draganov, and J. O. A. Robertsson, eds., 2008, Seismic interferometry: History and present status: SEG

Wapenaar, K., J. Thorbecke, D. Draganov, and J. Fokkema, 2002, Theory of acoustic daylight imaging revisited: 72nd Annual International Meeting, SEG Expanded Abstracts, 21, 2269.

Weaver, R. L., and O. I. Lobkis, 2001, Ultrasonics without a source: Thermal fluctuation correlations at MHz frequencies: Physical Review Letters, 87, 134301.

Yilmaz, O., 1999, Seismic data processing, 9th ed.: SEG. 